\title{
Ordering of Unicyclic Graphs with Perfect Matchings by Minimal Matching Energies
}

\author{
Jianming Zhu \\ Department of Applied Mathematics, Shanghai University of International Business and Economics, Shanghai, China \\ Email: zhujianming2005@suibe.edu.cn
}

How to cite this paper: Zhu, J.M. (2019) Ordering of Unicyclic Graphs with Perfect Matchings by Minimal Matching Energies. Open Journal of Discrete Mathematics, 9, 17-32.

https://doi.org/10.4236/ojdm.2019.91004

Received: December 28, 2018

Accepted: January 25, 2019

Published: January 28, 2019

Copyright $\odot 2019$ by author(s) and Scientific Research Publishing Inc. This work is licensed under the Creative Commons Attribution International License (CC BY 4.0).

http://creativecommons.org/licenses/by/4.0/

(c) (i) Open Access

\begin{abstract}
In 2012, Gutman and Wagner proposed the concept of the matching energy of a graph and pointed out that its chemical applications can go back to the 1970s. The matching energy of a graph is defined as the sum of the absolute values of the zeros of its matching polynomial. Let $u$ and $V$ be the non-isolated vertices of the graphs $G$ and $H$ with the same order, respectively. Let $w_{i}$ be a non-isolated vertex of graph $G_{i}$ where $i=1,2, \cdots, k$. We use $G_{u}(k)$ (respectively, $\left.H_{v}(k)\right)$ to denote the graph which is the coalescence of $G$ (respectively, $H$ ) and $G_{1}, G_{2}, \cdots, G_{k}$ by identifying the vertices $u$ (respectively, $v)$ and $w_{1}, w_{2}, \cdots, w_{k}$. In this paper, we first present a new technique of directly comparing the matching energies of $G_{u}(k)$ and $H_{v}(k)$, which can tackle some quasi-order incomparable problems. As the applications of the technique, then we can determine the unicyclic graphs with perfect matchings of order $2 n$ with the first to the ninth smallest matching energies for all $n \geq 211$.
\end{abstract}

\section{Keywords}

Matching Energy, Unicyclic Graph, Perfect Matching

\section{Introduction}

Let $G$ be a simple and undirected graph with $n$ vertices and $A(G)$ be its adjacency matrix. Let $\lambda_{1}, \lambda_{2}, \cdots, \lambda_{n}$ be the eigenvalues of $A(G)$. Then the energy of $G$, denoted by $E(G)$, is defined as [1]

$$
E(G)=\sum_{i=1}^{n}\left|\lambda_{i}\right|
$$

A fundamental problem encountered within the study of graph energy is the characterization of the graphs that belong to a given class of graphs having 
maximal or minimal energy, for example, Trees with extremal energies [2]-[15]; Unicyclic graphs with extremal energies [16]-[21]; Bicyclic graphs with extremal energies [22] [23] [24] [25]; Tricyclic graphs with extremal energies [26] [27] [28]. For more details, they can be found in the recent book [29] and review [30].

A matching in a graph $G$ is a set of pairwise nonadjacent edges. A matching is called $k$-matching if its size is $k$. Let $m(G, k)$ be the number of $k$-matching of $G$, where $m(G, k)=0$ for $k>\lfloor n / 2\rfloor$ or $k<0$. In addition, we assume that $m(G, 0)=1$.

The matching polynomial of a graph $G$ is defined as

$$
\alpha(G)=\alpha(G, x)=\sum_{k=0}^{\lfloor n / 2\rfloor}(-1)^{k} m(G, k) x^{n-2 k} .
$$

Recently, Gutman and Wagner [31] generalized the concept of graph energy and defined the matching energy of a graph $G$ based on the zeros of its matching polynomial.

Definition 1.1. Let $G$ be a simple graph of order $n$ and $\mu_{1}, \mu_{2}, \cdots, \mu_{n}$ be the zeros of its matching polynomial. Then

$$
\operatorname{ME}(G)=\sum_{i=1}^{n}\left|\mu_{i}\right|
$$

Further, Gutman and Wagner [31] pointed out that the matching energy is a quantity of relevance for chemical applications. They arrived at the simple relation:

$$
\operatorname{TRE}(G)=E(G)-M E(G),
$$

where $\operatorname{TRE}(G)$ is the so-called topological resonance energy of $G$, in connection with the chemical applications of matching energy, for more details see [32] [33] [34].

Similar to the integral formula for the energy of graph, Gutman and Wagner [31] have shown a beautiful integral formula for the matching energy of a graph $G$ as follows:

$$
M E(G)=\frac{2}{\pi} \int_{0}^{+\infty} \frac{1}{x^{2}} \ln \left(\sum_{k=0}^{\lfloor n / 2\rfloor} m(G, k) x^{2 k}\right) \mathrm{d} x .
$$

Then $M E(G)$ is a strictly monotonically increasing function of those numbers $m(G, k)(k=0,1, \cdots,\lfloor n / 2\rfloor)$. In the followings, the method of the quasi-order relation “ $\preceq$ ” is an important tool of comparing the matching energies of a pair of graphs.

Definition 1.2. Let $G_{1}$ and $G_{2}$ be two graphs of order n. If $m\left(G_{1}, k\right) \leq m\left(G_{2}, k\right)$ for all $k$ with $1 \leq k \leq\lfloor n / 2\rfloor$, then we write $G_{1} \preceq G_{2}$.

Furthermore, if $G_{1} \preceq G_{2}$ and there exists at least one index $j$ such that $m\left(G_{1}, j\right)<m\left(G_{2}, j\right)$, then we write $G_{1} \prec G_{2}$. If $m\left(G_{1}, k\right)=m\left(G_{2}, k\right)$ for all $k$, then we write $G_{1} \sim G_{2}$. According to the integral formula (1), we have for two graphs $G_{1}$ and $G_{2}$ of order $n$ that 


$$
\begin{aligned}
& G_{1} \preceq G_{2} \Rightarrow M E\left(G_{1}\right) \leq M E\left(G_{2}\right) \\
& G_{1} \prec G_{2} \Rightarrow M E\left(G_{1}\right)<M E\left(G_{2}\right) .
\end{aligned}
$$

In [31], Gutman and Wagner shown that its matching energy coincides with its energy if $T$ is a forest. Many properties of the matching energy are analogous to those of the graph energy. However, there are some notable differences. Then they raised a question: is it true that the matching energy of a graph $G$ coincides with its energy if and only if $G$ is a forest? Up to now, the question is still open.

The study on extremal matching energies is very interesting. In [31], Gutman and Wagner characterized the unicyclic graphs with the minimal and maximal matching energy. Zhu and Yang [35] determined the unicyclic graphs with the first eight minimal matching energies. In [36], Chen and Liu characterized the bipartite unicyclic graphs with the first $\lfloor(n-3) / 4\rfloor$ largest matching energies. Moreover, Chen et al. [37] determined the unicyclic odd-cycle graphs with the second to the fourth maximal matching energies. For bicyclic graph, Ji et al. [38] obtained the graphs with the minimal and maximal matching energy. In [39], Liu et al. further determined the bicyclic graphs with first five minimal matching energies and the second maximal matching energies, respectively. Chen and Shi [40] characterized tricyclic graph with maximal matching energy, for more results about extremal matching energies, see [41]-[47].

A fundamental problem encountered within the study of the matching energy is the characterization of the graphs that belong to a given class of graphs having maximal or minimal matching energy. One of the graph classes that are quite interestingly studied is the class of all unicyclic graphs with perfect matchings. As far as we are concerned, no results are on this topic. In this paper, we first present a new technique of directly comparing the matching energies of $G_{u}(k)$ and $H_{v}(k)$ in Section 2 (see Figure 2). As the applications of the technique, then we can determine the unicyclic graphs with perfect matchings of order $2 n$ with the first to the ninth smallest matching energies for all $n \geq 211$ in Section 3.

For simplicity, if $G_{1}$ is isomorphic to $G_{2}$, then we write $G_{1}=G_{2}$. If $G_{1}$ is not isomorphic to $G_{2}$, then we write $G_{1} \neq G_{2}$. Let $\mathbb{A}(2 n)$ be the set of the unicyclic graphs with perfect matchings of order $2 n$. Let the unicyclic graphs $A_{1}$, $A_{2}, A_{3}, A_{4}, A_{4}^{*}, A_{5}, A_{6}, A_{7}, A_{8}, A_{9}$ be shown in Figure 1. The following theorem is the main result of this paper.

Theorem 1.1. Let $G \in \mathbb{A}(2 n)$ and $n \geq 211$. If

$$
\begin{aligned}
& G \neq A_{1}, A_{2}, A_{3}, A_{4}, A_{4}^{*}, A_{5}, A_{6}, A_{7}, A_{8}, A_{9}, \text { then } \\
& \operatorname{ME}\left(A_{1}\right)<M E\left(A_{2}\right)<M E\left(A_{3}\right)<M E\left(A_{4}\right)=\operatorname{ME}\left(A_{4}^{*}\right)<M E\left(A_{5}\right) \\
& <M E\left(A_{6}\right)<M E\left(A_{7}\right)<M E\left(A_{8}\right)<M E\left(A_{9}\right)<M E(G)
\end{aligned}
$$

\section{A New Technique of Directly Comparing the Matching Energies of $G_{u}(k)$ and $H_{v}(k)$}

By Definition 1.2, we can see that the quasi-order method can be used to com- 
pare the matching energies of two graphs. However, if the quantities $m(G, k)$ cannot be compared uniformly, then the common comparing method is invalid, and this happens quite often. Recently much effort has been made to tackle these quasi-order incomparable problems [35] [39] [40].

Let $u$ and $v$ be the non-isolated vertices of the graphs $G$ and $H$ with the same order, respectively. Let $w_{i}$ be a non-isolated vertex of graph $G_{i}$ where $i=1,2, \cdots, k$. We use $G_{u}(k)$ (respectively, $\left.H_{v}(k)\right)$ to denote the graph which is the coalescence of $G$ (repectively, $H$ ) and $G_{1}, G_{2}, \cdots, G_{k}$ by identifying the vertices $u$ (respectively, $v$ ) and $w_{1}, w_{2}, \cdots, w_{k}$ (see Figure 2). In [14], He et al. presented a new method of directly comparing the energies of the bipartite graphs $G_{u}(k)$ and $H_{v}(k)$. In this section, we apply the main idea of this method to present a new technique of comparing the matching energies of the graphs $G_{u}(k)$ and $H_{v}(k)$ which can be used to tackle these quasi-order incomparable problems.

In this paper, we assume that

$$
\tilde{\alpha}(G)=\tilde{\alpha}(G, x)=\sum_{k=0}^{\lfloor n / 2\rfloor} m(G, k) x^{n-2 k} .
$$

By Equation (2), we can immediately obtain the following results.

Lemma 2.1. If two graphs $G$ and $H$ are disjoint, then

$$
\tilde{\alpha}(G \cup H)=\tilde{\alpha}(G) \cdot \tilde{\alpha}(H) .
$$

Lemma 2.2. ([35]) Let $G=(V(G), \mathcal{E}(G))$ be a graph. If $u$ is a vertex of $G$, then

$$
\tilde{\alpha}(G)=x \tilde{\alpha}(G-u)+\sum_{u v \in \mathcal{E}(G)} \tilde{\alpha}(G-u-v) .
$$

The coalescence of two graphs $G$ and $H$ with respect to vertex $u$ in $G$ and vertex $v$ in $H$, denoted by $G_{u} \cdot H_{v}$ (sometimes abbreviated as $G \cdot H$ ), is the graph obtained by identifying the vertices $u$ and $v$. Zhu and Yang [35] shown the recurrence relation of $\tilde{\alpha}(G \cdot H)$ in the following. For convenience of the reader, we present a full proof.

Lemma 2.3. ([35]) Let $G \cdot H$ be the coalescence of two graphs $G$ and $H$ with respect to vertex $u$ in $G$ and vertex $v$ in $H$. Then

$$
\tilde{\alpha}(G \cdot H)=\tilde{\alpha}(G) \tilde{\alpha}(H-v)+\tilde{\alpha}(G-u)(\tilde{\alpha}(H)-x \tilde{\alpha}(H-v)) .
$$

Proof. Using Lemmas 2.1 and 2.2, we can show

$$
\begin{aligned}
\tilde{\alpha}(G \cdot H)=x \tilde{\alpha}(G \cdot H-u)+\sum_{u w \in \mathcal{E}(G)} \tilde{\alpha}(G \cdot H-u-w)+\sum_{v t \in \mathcal{E}(H)} \tilde{\alpha}(G \cdot H-v-t) \\
=x \tilde{\alpha}(G-u) \cdot \tilde{\alpha}(H-v)+\sum_{u w \in \mathcal{E}(G)} \tilde{\alpha}(G-u-w) \cdot \tilde{\alpha}(H-v) \\
\quad+\sum_{v t \in \mathcal{E}(H)} \tilde{\alpha}(G-u) \cdot \tilde{\alpha}(H-v-t) \\
=\left(x \tilde{\alpha}(G-u)+\sum_{u w \in \mathcal{E}(G)} \tilde{\alpha}(G-u-w)\right) \cdot \tilde{\alpha}(H-v)+\tilde{\alpha}(G-u) \cdot \sum_{v \in \mathcal{E}(H)} \tilde{\alpha}(H-v-t) \\
=\tilde{\alpha}(G) \cdot \tilde{\alpha}(H-v)+\tilde{\alpha}(G-u) \cdot(\tilde{\alpha}(H)-x \tilde{\alpha}(H-v)) .
\end{aligned}
$$




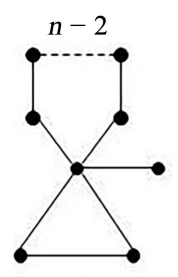

$A_{1}$

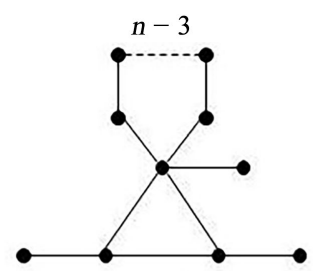

$A_{2}$

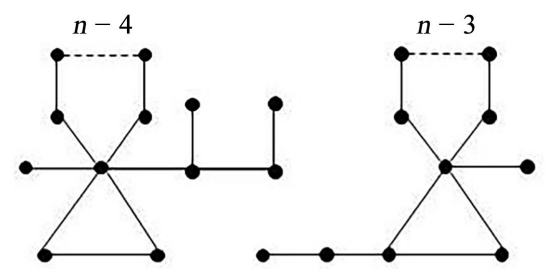

$A_{3}$
$A_{4}$

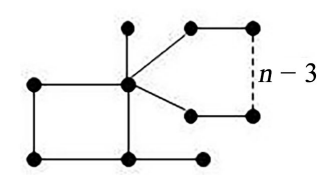

$A_{4}^{*}$

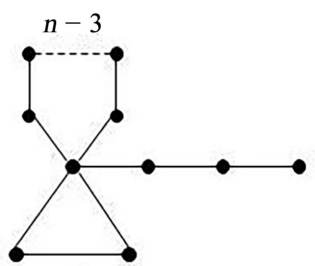

$A_{7}$

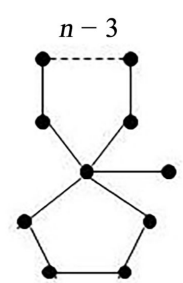

$A_{5}$

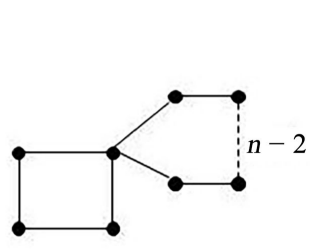

$A_{8}$

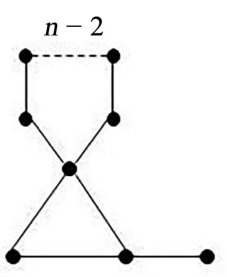

$A_{6}$

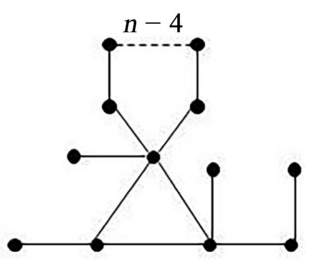

$A_{9}$

Figure 1. The graphs in $\mathbb{A}(2 n)$ with the first to the ninth smallest matching energies. For each graph $A_{i}$, the dashed lines denote the copies of $P_{3}$ attached to the maximal degree vertex.

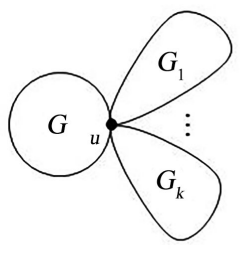

$G_{u}(k)$

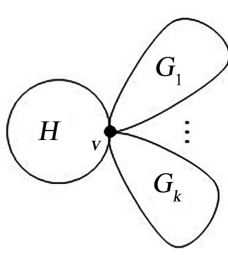

$H_{v}(k)$

Figure 2. The graphs $G_{u}(k)$ and $H_{v}(k)$.

From Lemma 2.3, we can get the recurrence relations of the graphs $\tilde{\alpha}\left(G_{u}(k)\right)$ and $\tilde{\alpha}\left(H_{v}(k)\right)$ which is a generalization of the formula for $\tilde{\alpha}(G \cdot H)$.

Lemma 2.4. Let $G_{u}(k)$ and $H_{v}(k)$ be defined as above (see Figure 2). Then we have the followings.
1) $\tilde{\alpha}\left(G_{u}(k)\right)=\prod_{i=1}^{k} \tilde{\alpha}\left(G_{i}-w_{i}\right)\left(\tilde{\alpha}(G)+\tilde{\alpha}(G-u)\left(\sum_{i=1}^{k} \frac{\tilde{\alpha}\left(G_{i}\right)}{\tilde{\alpha}\left(G_{i}-w_{i}\right)}-k x\right)\right)$;
2) $\tilde{\alpha}\left(H_{v}(k)\right)=\prod_{i=1}^{k} \tilde{\alpha}\left(G_{i}-w_{i}\right)\left(\tilde{\alpha}(H)+\tilde{\alpha}(H-v)\left(\sum_{i=1}^{k} \frac{\tilde{\alpha}\left(G_{i}\right)}{\tilde{\alpha}\left(G_{i}-w_{i}\right)}-k x\right)\right)$.

Proof. 1) We prove the result by induction on $k$. When $k=1$, by Lemma 2.3 we have 


$$
\tilde{\alpha}\left(G_{u}(1)\right)=\tilde{\alpha}\left(G \cdot G_{1}\right)=\tilde{\alpha}(G) \tilde{\alpha}\left(G_{1}-w_{1}\right)+\tilde{\alpha}(G-u)\left(\tilde{\alpha}\left(G_{1}\right)-x \tilde{\alpha}\left(G_{1}-w_{1}\right)\right),
$$

which implies that the result holds. We assume that the result holds for $k-1$ in what follows. For simplicity, we write $h_{k}=\sum_{i=1}^{k} \frac{\tilde{\alpha}\left(G_{i}\right)}{\tilde{\alpha}\left(G_{i}-w_{i}\right)}-k x$. By Lemmas 2.1 and 2.3 , we can show

$$
\begin{aligned}
& \tilde{\alpha}\left(G_{u}(k)\right)=\tilde{\alpha}\left(G_{u}(k-1) \cdot G_{k}\right) \\
&= \tilde{\alpha}\left(G_{u}(k-1)\right) \tilde{\alpha}\left(G_{k}-w_{k}\right)+\tilde{\alpha}\left(G_{u}(k-1)-u\right)\left(\tilde{\alpha}\left(G_{k}\right)-x \tilde{\alpha}\left(G_{k}-w_{k}\right)\right) \\
&= \prod_{i=1}^{k-1} \tilde{\alpha}\left(G_{i}-w_{i}\right)\left(\tilde{\alpha}(G)+\tilde{\alpha}(G-u) h_{k-1}\right) \tilde{\alpha}\left(G_{k}-w_{k}\right) \\
&+\prod_{i=1}^{k-1} \tilde{\alpha}\left(G_{i}-w_{i}\right) \tilde{\alpha}(G-u)\left(\tilde{\alpha}\left(G_{k}\right)-x \tilde{\alpha}\left(G_{k}-w_{k}\right)\right) \\
&=\prod_{i=1}^{k-1} \tilde{\alpha}\left(G_{i}-w_{i}\right)\left(\left(\tilde{\alpha}(G)+\tilde{\alpha}(G-u) h_{k-1}\right) \tilde{\alpha}\left(G_{k}-w_{k}\right)\right. \\
&+\left.\tilde{\alpha}(G-u)\left(\tilde{\alpha}\left(G_{k}\right)-\tilde{\alpha}\left(G_{k}-w_{k}\right)\right)\right) \\
&=\prod_{i=1}^{k-1} \tilde{\alpha}\left(G_{i}-w_{i}\right) \tilde{\alpha}\left(G_{k}-w_{k}\right)\left(\tilde{\alpha}(G)+\tilde{\alpha}(G-u) h_{k-1}+\tilde{\alpha}(G-u)\left(\frac{\tilde{\alpha}\left(G_{k}\right)}{\tilde{\alpha}\left(G_{k}-w_{k}\right)}-x\right)\right) \\
&=\prod_{i=1}^{k} \tilde{\alpha}\left(G_{i}-w_{i}\right)\left(\tilde{\alpha}(G)+\tilde{\alpha}(G-u) h_{k-1}+\tilde{\alpha}(G-u)\left(\frac{\tilde{\alpha}\left(G_{k}\right)}{\tilde{\alpha}\left(G_{k}-w_{k}\right)}-x\right)\right) \\
&=\prod_{i=1}^{k} \tilde{\alpha}\left(G_{i}-w_{i}\right)\left(\tilde{\alpha}(G)+\tilde{\alpha}(G-u)\left(h_{k-1}+\frac{\tilde{\alpha}\left(G_{k}\right)}{\tilde{\alpha}\left(G_{k}-w_{k}\right)}-x\right)\right) \\
&=\prod_{i=1}^{k} \tilde{\alpha}\left(G_{i}-w_{i}\right)\left(\tilde{\alpha}(G)+\tilde{\alpha}(G-u) h_{k}\right)
\end{aligned}
$$

Then we can see that the result holds.

2) The proof is similar to 1 ).

The following lemma illustrates an integral formula for the difference of the matching energies of two graphs with the same order which was obtained by Zhu and Yang [35].

Lemma 2.5. ([35]) Let $\alpha(G, x)$ and $\alpha(H, x)$ be the matching polynomials of two graphs $G$ and $H$ with the same order, respectively. Then

$$
M E(G)-M E(H)=\frac{2}{\pi} \int_{0}^{+\infty} \ln \frac{\tilde{\alpha}(G, x)}{\tilde{\alpha}(H, x)} \mathrm{d} x .
$$

Let $x>0$. For simplicity, we write

$$
h_{k}=\sum_{i=1}^{k} \frac{\tilde{\alpha}\left(G_{i}\right)}{\tilde{\alpha}\left(G_{i}-w_{i}\right)}-k x=\sum_{i=1}^{k} \frac{\tilde{\alpha}\left(G_{i}\right)-x \tilde{\alpha}\left(G_{i}-w_{i}\right)}{\tilde{\alpha}\left(G_{i}-w_{i}\right)} .
$$

From Lemma 2.2, we have $h_{k}>0$ and $h_{l}<h_{k}$ holds for any positive integer $l<k$.

In what follows, we define two sets $M$ and $M^{c}$ as follows:

$$
M=\{x>0 \mid \tilde{\alpha}(G-u) \tilde{\alpha}(H)-\tilde{\alpha}(G) \tilde{\alpha}(H-v)>0\}
$$




$$
M^{c}=\{x>0 \mid \tilde{\alpha}(G-u) \tilde{\alpha}(H)-\tilde{\alpha}(G) \tilde{\alpha}(H-v) \leq 0\} .
$$

It is easily checked that $M \bigcup M^{c}=(0,+\infty)$. Furthermore, we write

$$
\begin{gathered}
m_{k}(x)=\frac{\tilde{\alpha}(G)+h_{k} \tilde{\alpha}(G-u)}{\tilde{\alpha}(H)+h_{k} \tilde{\alpha}(H-v)} \\
m(x)=\frac{\tilde{\alpha}(G-u)}{\tilde{\alpha}(H-v)} .
\end{gathered}
$$

Combining Lemma 2.4 with Lemma 2.5, we can present a new technique for directly comparing the matching energies of two graphs $G_{u}(k)$ and $H_{v}(k)$ in the following theorem.

Theorem 2.2. Let $M, M^{c}, m_{k}(x)$ and $m(x)$ be defined as above. For all positive integers $1 \leq l<k$, we have

$$
\begin{aligned}
\int_{M} \ln m_{l}(x) \mathrm{d} x+\int_{M^{c}} \ln m(x) \mathrm{d} x & \leq \frac{\pi}{2}\left(M E\left(G_{u}(k)\right)-M E\left(H_{v}(k)\right)\right) \\
& \leq \int_{M} \ln m(x) \mathrm{d} x+\int_{M^{c}} \ln m_{l}(x) \mathrm{d} x .
\end{aligned}
$$

Proof. By some calculations, we can obtain that

$$
\begin{aligned}
m_{k}(x)-m_{l}(x)=\frac{\tilde{\alpha}(G)+h_{k} \tilde{\alpha}(G-u)}{\tilde{\alpha}(H)+h_{k} \tilde{\alpha}(H-v)}-\frac{\tilde{\alpha}(G)+h_{l} \tilde{\alpha}(G-u)}{\tilde{\alpha}(H)+h_{l} \tilde{\alpha}(H-v)} \\
=\frac{\left(h_{k}-h_{l}\right)(\tilde{\alpha}(G-u) \tilde{\alpha}(H)-\tilde{\alpha}(G) \tilde{\alpha}(H-v))}{\left(\tilde{\alpha}(H)+h_{k} \tilde{\alpha}(H-v)\right)\left(\tilde{\alpha}(H)+h_{l} \tilde{\alpha}(H-v)\right)} . \\
m_{k}(x)-m(x)=\frac{\tilde{\alpha}(G)+h_{k} \tilde{\alpha}(G-u)}{\tilde{\alpha}(H)+h_{k} \tilde{\alpha}(H-v)}-\frac{\tilde{\alpha}(G-u)}{\tilde{\alpha}(H-v)} \\
=\frac{-(\tilde{\alpha}(G-u) \tilde{\alpha}(H)-\tilde{\alpha}(G) \tilde{\alpha}(H-v))}{\left(\tilde{\alpha}(H)+h_{k} \tilde{\alpha}(H-v)\right) \tilde{\alpha}(H-v)} .
\end{aligned}
$$

Thus, If $x \in M$, then $m_{l}(x) \leq m_{k}(x) \leq m(x)$. If $x \in M^{c}$, then $m(x) \leq m_{k}(x) \leq m_{l}(x)$.

Moreover, by Lemmas 2.4 and 2.5, we have

$$
\begin{aligned}
& \frac{\pi}{2}\left(M E\left(G_{u}(k)\right)-M E\left(H_{v}(k)\right)\right) \\
& =\int_{0}^{+\infty} \ln m_{k}(x) \mathrm{d} x=\int_{M} \ln m_{k}(x) \mathrm{d} x+\int_{M^{c}} \ln m_{k}(x) \mathrm{d} x .
\end{aligned}
$$

Then the result can be obtained immediately.

Next, we use the new technique to compare the matching energies of the quasi-order incomparable graphs $A_{5}$ and $A_{6}, A_{8}$ and $A_{9}$ (see Figure 1), respectively. Denote by $C_{k}$ and $P_{k}$ the cycle of length $k$ and the path of length $k-1$, respectively.

Lemma 2.6. If $n \geq 6$, then $\operatorname{ME}\left(A_{5}\right)<M E\left(A_{6}\right)$.

Proof. Let $G$ be the graph obtained by attaching a pendent edge to a vertex $u$ of $C_{5}$. Let $H$ be the graph obtained by attaching a pendent edge and a pendent path of length 2 to the vertices $W$ and $V$ of $C_{3}$, respectively. Let $G_{1}=G_{2}=\cdots=G_{n-3}=P_{3}$ and $w_{i}$ be the pendent vertex of $G_{i}$. Then 
$G_{u}(n-3)=A_{5}$ and $H_{v}(n-3)=A_{6}$ (see Figure 1$)$. By some calculations, we can show

$$
\begin{gathered}
\tilde{\alpha}(G)=x^{6}+6 x^{4}+8 x^{2}+1 \\
\tilde{\alpha}(G-u)=x\left(x^{4}+3 x^{2}+1\right) \\
\tilde{\alpha}(H)=x^{6}+6 x^{4}+7 x^{2}+1 \\
\tilde{\alpha}(H-v)=\left(x^{2}+1\right)\left(x^{3}+2 x\right) .
\end{gathered}
$$

It follows that

$$
\tilde{\alpha}(G-u) \tilde{\alpha}(H)-\tilde{\alpha}(G) \tilde{\alpha}(H-v)=-2 x^{7}-9 x^{5}-9 x^{3}-x .
$$

This implies that $M=\varnothing$ and $M^{c}=(0,+\infty)$. By Theorem 2.2 and some calculations using the software MATLAB, we have

$$
\begin{aligned}
& \frac{\pi}{2}\left(M E\left(A_{5}\right)-M E\left(A_{6}\right)\right) \\
& =\frac{\pi}{2}\left(M E\left(G_{u}(n-3)\right)-M E\left(H_{v}(n-3)\right)\right) \\
& \leq \int_{0}^{+\infty} \ln m_{3}(x) \mathrm{d} x \\
& =\int_{0}^{+\infty} \ln \frac{\left(x^{2}+1\right)^{2}\left(x^{8}+10 x^{6}+23 x^{4}+12 x^{2}+1\right)}{\left(x^{2}+1\right)^{3}\left(x^{6}+9 x^{4}+13 x^{2}+1\right)} \mathrm{d} x \\
& \doteq-0.0248<0 .
\end{aligned}
$$

Thus, $\operatorname{ME}\left(A_{5}\right)<\operatorname{ME}\left(A_{6}\right)$.

Lemma 2.7. If $n \geq 211$, then $M E\left(A_{8}\right)<M E\left(A_{9}\right)$.

Proof. Let $G$ be the graph obtained by attaching two pendent paths of length 2 to the same vertex of $C_{4}$. Let $H$ be the graph obtained by first attaching a pendent edge to each vertex of $C_{3}$ and then attaching a pendent path of length 2 to one vertex of $C_{3}$. Let $u$ be the vertex of degree 4 in $G$ and $v$ be the vertex of degree 3 in $H$, respectively. Let $G_{1}=G_{2}=\cdots=G_{n-4}=P_{3}$ and $w_{i}$ be the pendent vertex of $G_{i}$. Then $G_{u}(n-4)=A_{8}$ and $H_{v}(n-4)=A_{9}$ (see Figure 1). By some calculations, we can get the followings.

$$
\begin{gathered}
\tilde{\alpha}(G)=x^{8}+8 x^{6}+17 x^{4}+12 x^{2}+2 \\
\tilde{\alpha}(G-u)=\left(x^{2}+1\right)^{2}\left(x^{3}+2 x\right) \\
\tilde{\alpha}(H)=x^{8}+8 x^{6}+15 x^{4}+8 x^{2}+1 \\
\tilde{\alpha}(H-v)=x\left(x^{6}+5 x^{4}+5 x^{2}+1\right),
\end{gathered}
$$

which implies that

$$
\tilde{\alpha}(G-u) \tilde{\alpha}(H)-\tilde{\alpha}(G) \tilde{\alpha}(H-v)=-x^{3}\left(x^{2}+1\right)^{2}\left(x^{6}+8 x^{4}+11 x^{2}+1\right) .
$$

It follows that $M=\varnothing$ and $M^{c}=(0,+\infty)$. By Theorem 2.2 and some calculations using the software MATLAB, we have 


$$
\begin{aligned}
& \frac{\pi}{2}\left(M E\left(A_{8}\right)-M E\left(A_{9}\right)\right) \\
& =\frac{\pi}{2}\left(M E\left(G_{u}(n-4)\right)-M E\left(H_{v}(n-4)\right)\right) \\
& \leq \int_{0}^{+\infty} \ln m_{207}(x) \mathrm{d} x \\
& =\int_{0}^{+\infty} \ln \frac{\left(x^{2}+1\right)^{208}\left(x^{6}+214 x^{4}+424 x^{2}+2\right)}{\left(x^{2}+1\right)^{206}\left(x^{10}+216 x^{8}+1055 x^{6}+1055 x^{4}+216 x^{2}+1\right)} \mathrm{d} x \\
& \doteq-7.43 \times 10^{-5}<0 .
\end{aligned}
$$

Consequently, $\operatorname{ME}\left(A_{8}\right)<M E\left(A_{9}\right)$.

\section{Minimal Matching Energies of Unicyclic Graphs with Perfect Matchings of Order $2 n$}

In this section, we will determine the unicyclic graphs with perfect matchings of order $2 n$ with the first to the ninth smallest matching energies (i.e., to prove Theorem 1.1).

In what follows, we denote by $M(G)$ a perfect matching of a graph $G$. Let $\hat{G}=G-M(G)-S_{0}$, where $S_{0}$ is the set of isolated vertices in $G-M(G)$. We call $\hat{G}$ the capped graph of $G$ and $G$ the original graph of $\hat{G}$. For example, the capped graphs of $A_{1}, A_{2}, A_{3}, A_{5}$ are shown in Figure 3.

Let $G \in \mathbb{A}(2 n)$. Denote by $\mathcal{E}(G)$ the edge set of $G$. It is easy to see that $\mathcal{E}(G)=\mathcal{E}(\hat{G}) \cup M(G)$. Thus each $k$-matching $\Omega$ of $G$ can be partitioned into two parts: $\Omega=\Phi \bigcup \Psi$, where $\Phi \subseteq \mathcal{E}(\hat{G})$ and $\Psi \subseteq M(G)$. Let $r_{j}^{(2 k)}(G)$ be the number of ways to choose $k$ independent edges in $G$ such that just $j$ edges are in $\hat{G}$. We agree that $r_{0}^{(0)}(G)=1$ and $r_{j}^{(2 k)}(G)=0(k<0)$. For example: $r_{0}^{(2 k)}(G)=\left(\begin{array}{l}n \\ k\end{array}\right)$ and $r_{1}^{(2 k)}(G)=n\left(\begin{array}{c}n-2 \\ k-1\end{array}\right)$.

Then we have

$$
m(G, k)=\sum_{j=0}^{k} r_{j}^{(2 k)}(G)=p+\sum_{j=2}^{k} r_{j}^{(2 k)}(G)
$$

where

$$
p=\left(\begin{array}{l}
n \\
k
\end{array}\right)+n\left(\begin{array}{l}
n-2 \\
k-1
\end{array}\right) .
$$

This is the main method to compute $m(G, k)$ of a graph $G$ in what follows.

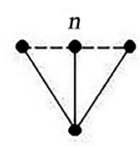

$\hat{A_{1}}$

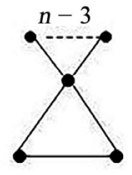

$\hat{A}_{2}$

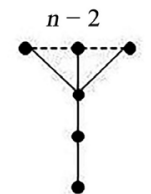

$\hat{A}_{3}$

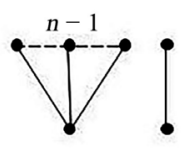

$\hat{A}_{5}$

Figure 3. The capped graphs of $A_{1}, A_{2}, A_{3}$ and $A_{5}$. For each graph, the dashed lines denote the copies of $P_{2}$ attached to the maximal degree vertex. 
Let $X_{n}$ be the star of order $n$. Let $Y_{n}$ be the graph of order $n$ obtained by attaching $n-3$ pendent edges to a pendent vertex of $P_{3}$. Let $Z_{n}$ be the graph of order $n$ obtained from $P_{4}=v_{1} v_{2} v_{3} v_{4}$ by attaching $n-5$ and one pendent edges to $v_{2}$ and $v_{3}$, respectively. In [2] and [31], the following results were shown.

Lemma 3.1. ([2]) Let $T$ be a tree of order $n \geq 5$. Then $m\left(X_{n}, k\right) \leq m\left(Y_{n}, k\right) \leq m\left(Z_{n}, k\right) \leq m(T, k)$, and the equalities do not hold for all $k$, where $T \neq X_{n}, Y_{n}, Z_{n}$ and $0 \leq k \leq\lfloor n / 2\rfloor$.

Lemma 3.2. ([31]) Suppose that $G$ is a connected graph and $T$ is an induced subgraph of $G$ such that $T$ is a tree and $T$ is connected to the rest of $G$ only by a cut vertex $v$. If $T$ is replaced by a star of the same order, centered at $v$, then the quasi-order decreases (unless $T$ is already such a star).

Let $S_{n}^{l}$ be the unicyclic graph of order $n$ obtained by attaching $n-l$ pendent edges to one vertex of $C_{l}$.

Lemma 3.3. ([43]) Let $G$ be a unicyclic graph of order $n$ with a cycle of length l. If $G \neq S_{n}^{l}$, then $G \succ S_{n}^{l}$.

Let $R_{n}^{3}$ be the graph of order $n$ obtained by attaching $n-4$ and one pendent edges to $v_{1}$ and $v_{2}$ of $C_{3}=v_{1} v_{2} v_{3} v_{1}$, respectively. Let $C_{3}(a, b, c)$ be the unicyclic graph obtained by attaching $a, b, c$ pendent edges to $v_{1}, v_{2}, v_{3}$ of $C_{3}=v_{1} v_{2} v_{3} v_{1}$, respectively. Let $Q_{n}^{3}$ be the graph of order $n$ obtained by attaching $n-4$ pendent edges to the pendent vertex of $C_{3}(1,0,0)$. The graphs $S_{n}^{3}$, $R_{n}^{3}$ and $Q_{n}^{3}$ are shown in Figure 4.

Lemma 3.4. Let $G$ be a unicyclic graph of order $n \geq 9$. If $G \neq S_{n}^{3}, R_{n}^{3}$, then $m(G, 2) \geq 2 n-6$.

Proof. Let $G$ be a unicyclic graph with the unique cycle of length $l$. We consider the following cases.

Case 1: $l \geq 5$.

By Lemma 3.10, we have $G \succeq S_{n}^{l}$. Then $m(G, 2) \geq m\left(S_{n}^{l}, 2\right) \geq(n-l)(l-2) \geq 3(n-5) \geq 2 n-6$.

Case 2: $l=4$.

Using Lemma 3.10, we can show $G \succeq S_{n}^{4}$. So, $m(G, 2) \geq m\left(S_{n}^{4}, 2\right)=2 n-6$.

Case 3: $l=3$.

Denote by $d_{G}(u)$ the degree of the vertex $u$ in $G$. Let $C_{3}=v_{1} v_{2} v_{3} v_{1}$ be the unique cycle of the unicyclic graph $G$ and $N(G)=\left\{v_{i} \mid d_{G}\left(v_{i}\right) \geq 3, i=1,2,3\right\}$.

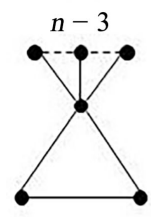

$S_{n}^{3}$

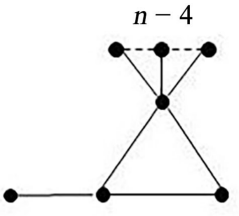

$R_{n}^{3}$

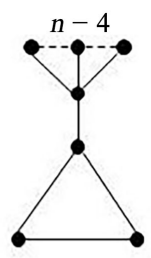

$Q_{n}^{3}$

Figure 4. The graphs $S_{n}^{3}, R_{n}^{3}$ and $Q_{n}^{3}$ in Lemma 3.4. For each graph, the dashed lines denote the copies of $P_{2}$ attached to the maximal degree vertex. 
Subcase 3.1: $|N(G)|=1$.

Without loss of generality, we can assume that $d_{G}\left(v_{1}\right) \geq 3$. Let $T$ be the rooted tree of order $n-2$ with the root $v_{1}$ in $G$. If $T=X_{n-2}$, then $G=Q_{n}^{3}$ $\left(G \neq S_{n}^{3}\right)$. Then $m(G, 2)=3 n-11>2 n-6$. If $T=Y_{n-2}$, then $m(G, 2) \geq(n-3)+m\left(Y_{n-2}, 2\right)+2=(n-3)+(n-5)+2=2 n-6$. If $T \neq X_{n-2}, Y_{n-2}$, then by Lemma 3.8 we have

$m(G, 2) \geq(n-3)+m(T, 2) \geq(n-3)+m\left(Z_{n-2}, 2\right)=n-3+2(n-6)=3 n-15 \geq 2 n-6$.

Subcase 3.2: $|N(G)|=2$.

Without loss of generality, we can assume that $d_{G}\left(v_{1}\right) \geq 3$ and $d_{G}\left(v_{2}\right) \geq 3$. Let $T_{1}$ and $T_{2}$ be the rooted tree with the root $v_{1}$ and $v_{2}$ in $G$, respectively.

If $T_{1}=P_{2}$ or $T_{2}=P_{2}$, then by Lemma 3.9 we can show $m(G, 2) \geq m\left(R_{n}^{3}, 2\right)=2 n-7$. Since $G \neq R_{n}^{3}$, we have $m(G, 2) \geq 2 n-6$.

If $T_{1} \neq P_{2}$ and $T_{2} \neq P_{2}$, then by Lemma 3.9 we have $G \succeq C_{3}(a, b, 0)$ $(a+b=n-3)$. Thus, $m(G, 2) \geq m\left(C_{3}(a, b, 0), 2\right) \geq a+b+a b \geq n-3+2(n-5)=3 n-13>2 n-6$.

Subcase 3.3: $|N(G)|=3$.

According to Lemma 3.9, we have $G \succeq C_{3}(a, b, c)(a+b+c=n-3)$. Then we have

$$
\begin{aligned}
m(G, 2) & \geq a+b+c+a b+b c+a c \\
& \geq n-3+a+b-1+b+c-1+a+c-1 \\
& =n-3+2(n-3)-3 \\
& =3 n-12>2 n-6 .
\end{aligned}
$$

Thus we have completed the proof.

Lemma 3.5. Let $G \in \mathbb{A}(2 n)$ and $n \geq 9$. If

$G \neq A_{1}, A_{2}, A_{3}, A_{4}, A_{4}^{*}, A_{5}, A_{6}, A_{7}, A_{8}, A_{9}$, then $m(\hat{G}, 2) \geq 2 n-6$.

Proof. We consider the following cases.

Case 1: $\hat{G}$ is a connected graph.

Subcase 1.1: $\hat{G}$ is a tree.

It can easily be verified that $G=A_{1}$ as $\hat{G}=X_{n+1}$ and $G=A_{3}, A_{4}, A_{4}^{*}, A_{6}$ as $\hat{G}=Y_{n+1}$. Thus, $\hat{G} \neq X_{n+1}, Y_{n+1}$. By Lemma 3.8, we have $m(\hat{G}, 2) \geq m\left(Z_{n+1}, 2\right)=2 n-6$.

Subcase 1.2: $\hat{G}$ is a connected unicyclic graph.

It can be shown that $G=A_{2}$ as $\hat{G}=S_{n}^{3}$ and $G=A_{9}$ as $\hat{G}=R_{n}^{3}$. Therefore, $\hat{G} \neq S_{n}^{3}, R_{n}^{3}$. According to Lemma 3.11, we have $m(\hat{G}, 2) \geq 2 n-6$.

Case 2: $\hat{G}$ is a unconnected graph.

Subcase 2.1: $\hat{G}$ is only composed of trees.

It can be checked that $G=A_{5}, A_{7}, A_{8}$ as $\hat{G}=X_{n} \cup P_{2}$. Then, $\hat{G} \neq X_{n} \cup P_{2}$. Let $\hat{G}_{1}$ be the coalescence of all trees in a way such that $\hat{G}_{1} \neq X_{n+1}, Y_{n+1}$. It is clear that $m(\hat{G}, 2)>m\left(\hat{G}_{1}, 2\right)$. Similar to Subcase 1.1, we have $m(\hat{G}, 2)>2 n-6$.

Subcase 2.2: $\hat{G}$ is composed of trees and unicyclic graphs.

Let $\hat{G}_{2}$ be the coalescence of all trees and unicyclic graphs in a way such that $\hat{G}_{2} \neq S_{n}^{3}, R_{n}^{3}$. It is obvious that $m(\hat{G}, 2)>m\left(\hat{G}_{2}, 2\right)$. Similar to Subcase 1.2 , we 
have $m(\hat{G}, 2)>2 n-6$.

Then we have completed the proof.

From Lemma 3.5, we can immediately derive the following result.

Lemma 3.6. Let $G \in \mathbb{A}(2 n)$ and $n \geq 9$. If $G \neq A_{1}, A_{2}, A_{3}, A_{4}, A_{4}^{*}, A_{5}, A_{6}, A_{7}, A_{8}, A_{9}$, then $G \succ A_{9}$.

Proof. By Equation (3) and Lemma 3.12, when $k \geq 2$, we can get

$$
\begin{aligned}
m(G, k) & =p+\sum_{j=2}^{k} r_{j}^{(2 k)}(G) \geq p+r_{2}^{(2 k)}(G) \\
& \geq p+m(\hat{G}, 2)\left(\begin{array}{l}
n-4 \\
k-2
\end{array}\right) \geq p+(2 n-6)\left(\begin{array}{l}
n-4 \\
k-2
\end{array}\right) .
\end{aligned}
$$

Furthermore, by some calculations we have

$$
m\left(A_{9}, k\right)=p+(2 n-7)\left(\begin{array}{l}
n-4 \\
k-2
\end{array}\right) .
$$

Then we can see that $G \succ A_{9}$.

Combining Lemma 2.6 with Lemma 2.7, we can show the followings.

Lemma 3.7. If $n \geq 211$, then

$$
\begin{aligned}
M E\left(A_{1}\right) & <M E\left(A_{2}\right)<M E\left(A_{3}\right)<M E\left(A_{4}\right)=\operatorname{ME}\left(A_{4}^{*}\right)<M E\left(A_{5}\right) . \\
& <M E\left(A_{6}\right)<M E\left(A_{7}\right)<M E\left(A_{8}\right)<M E\left(A_{9}\right)
\end{aligned} .
$$

Proof. Using Equation (4) and some calculations, we can get

$$
\begin{gathered}
m\left(A_{1}, k\right)=p \\
m\left(A_{2}, k\right)=p+(n-3)\left(\begin{array}{l}
n-4 \\
k-2
\end{array}\right) \\
m\left(A_{3}, k\right)=p+(n-2)\left(\begin{array}{l}
n-4 \\
k-2
\end{array}\right) \\
m\left(A_{4}, k\right)=p+\left(\begin{array}{l}
n-3 \\
k-2
\end{array}\right)+(n-3)\left(\begin{array}{l}
n-4 \\
k-2
\end{array}\right) \\
m\left(A_{4}^{*}, k\right)=p+\left(\begin{array}{l}
n-3 \\
k-2
\end{array}\right)+(n-3)\left(\begin{array}{l}
n-4 \\
k-2
\end{array}\right) \\
m\left(A_{5}, k\right)=p+2\left(\begin{array}{l}
n-3 \\
k-2
\end{array}\right)+(n-3)\left(\begin{array}{l}
n-4 \\
k-2
\end{array}\right) \\
m\left(A_{6}, k\right)=p+(n-3)\left(\begin{array}{l}
n-3 \\
k-2
\end{array}\right) \\
m\left(A_{7}, k\right)=p+(n-1)\left(\begin{array}{l}
n-3 \\
k-2
\end{array}\right) \\
m\left(A_{8}, k\right)=p+\left(\begin{array}{l}
n-2 \\
k-2
\end{array}\right)+\left(\begin{array}{l}
n-2
\end{array}\right)\left(\begin{array}{l}
n-3 \\
k-2
\end{array}\right) \\
m\left(A_{9}, k\right)=p+(2 n-7)\left(\begin{array}{l}
n-4 \\
k-2
\end{array}\right) .
\end{gathered}
$$


It implies that $A_{1} \prec A_{2} \prec A_{3} \prec A_{4} \prec A_{5}$ and $A_{6} \prec A_{7} \prec A_{8}$. From Lemmas 2.6 and 2.7 , the result can be easily obtained.

\section{Proof of Theorem 1.1:}

Proof. The result can follow immediately by Lemmas 3.13 and 3.14 .

\section{Conclusions}

In this paper, we first present a new technique of directly comparing the matching energies of $G_{u}(k)$ and $H_{v}(k)$, which can tackle some quasi-order incomparable problems. As the applications of the technique, we then determine the unicyclic graphs with perfect matchings of order $2 n$ with the first to the ninth smallest matching energies for all $n \geq 211$. Furthermore, we can consider characterizing the extremal graphs with maximal or minimal matching energy for other classes of graphs, e.g. graphs with different parameters. These are our work in the future.

The results presented in this paper are for a fixed graph. In reality, most of the graphs or networks are evolving. Some graph invariants have been studied in this setting, e.g. the Estrada index of evolving graphs [48]; Laplacian Estrada and normalized Laplacian Estrada indices of evolving graphs [49]. Then we can consider studying the matching energy of evolving graphs in the future.

\section{Acknowledgements}

We thank the editor and the referee for their valuable comments. This work is supported by the National Natural Science Foundation of China (No. 11501356) and (No. 11426149).

\section{Conflicts of Interest}

The author declares no conflicts of interest regarding the publication of this paper.

\section{References}

[1] Gutman, I. (1978) The Energy of a Graph. Berichte Mathematisch-statistische Sektion Forschungszentrum Graz, 103, 1-22.

[2] Gutman, I. (1977) Acyclic Systems with Extremal Hukel-Electron Energy. Theoretica Chimica Acta, 45, 79-87. https://doi.org/10.1007/BF00552542

[3] Li, N. and Li, S. (2008) On the Extremal Energy of Trees. MATCH Communications in Mathematical and in Computer Chemistry, 59, 291-314.

[4] Gutman, I., Radenkovic, S., Li, N. and Li, S. (2008) Extremal Energy of Trees. MATCH Communications in Mathematical and in Computer Chemistry, 59, 315-320.

[5] Wang, W. and Kang, L. (2010) Ordering of the Trees by Minimal Energy. Journal of Mathematical Chemistry, 47, 937-958. https://doi.org/10.1007/s10910-009-9616-3

[6] Shan, H. and Shao, J. (2010) Graph Energy Change Due to Edge Grafting Operations and Its Applications. MATCH Communications in Mathematical and in Computer Chemistry, 64, 25-40.

[7] Huo, B., Ji, S., Li, X. and Shi, Y. (2011) Complete Solution to a Conjecture on the 
Fourth Maximal Energy Tree. MATCH Communications in Mathematical and in Computer Chemistry, 66, 903-912.

[8] Shan, H. and Shao, J. (2012) The Proof of a Conjecture on the Comparison of the Energies of Trees. Journal of Mathematical Chemistry, 50, 2637-2647.

https://doi.org/10.1007/s10910-012-0052-4

[9] Andriantiana, E.O.D. (2012) More Trees with Large Energy. MATCH Communications in Mathematical and in Computer Chemistry, 68, 675-695.

[10] Shan, H., Shao, J., Zhang, L. and He, C. (2012) A New Method of Comparing the Energies of Subdivision Bipartite Graphs. MATCH Communications in Mathematical and in Computer Chemistry, 68, 721-740.

[11] Shan, H., Shao, J., Zhang, L. and He, C. (2012) Proof of a Conjecture on Trees with Lagre Energy. MATCH Communications in Mathematical and in Computer Chemistry, 68, 703-720.

[12] Gutman, I., Furtula, B., Andriantiana, E.O.D. and Cvetic, M. (2012) More Trees with Large Energy and Small Size. MATCH Communications in Mathematical and in Computer Chemistry, 68, 697-702.

[13] MArn, C., Monsalve, J. and Rada, J. (2015) Maximum and Minimum Energy Trees with Two and Three Branched Vertices. MATCH Communications in Mathematical and in Computer Chemistry, 74, 285-306.

[14] He, C., Lei, L., Shan, H. and Peng, A. (2017) Two Subgraph Grafting Theoerms on the Energy of Bipartite Graphs. Linear Algebra and its Applications, 515, 96-110. https://doi.org/10.1016/j.laa.2016.11.010

[15] Zhu, J. and Yang, J. (2018) Minimal Energies of Trees with Three Branched Vertices. MATCH Communications in Mathematical and in Computer Chemistry, 79, 263-274.

[16] Hou, Y. (2001) Unicyclic Graphs with Minimal Energy. Journal of Mathematical Chemistry, 29, 163-168. https://doi.org/10.1023/A:1010935321906

[17] Hou, Y., Gutman, I. and Woo, C. (2002) Unicyclic Graphs with Maximal Energy. Linear Algebra and Its Applications, 356, 27-36. https://doi.org/10.1016/S0024-3795(01)00609-7

[18] Andriantiana, E.O.D. (2011) Unicylic Bipartite Graphs with Maximum Energy. MATCH Communications in Mathematical and in Computer Chemistry, 66, 913-926.

[19] Huo, B., Li, X. and Shi, Y. (2011) Complete Solution to a Conjecture on the Maximal Energy of Unicyclic Graphs. European Journal of Combinatorics, 32, 662-673. https://doi.org/10.1016/j.ejc.2011.02.011

[20] Wang, W. (2011) Ordering of Unicyclic Graphs with Perfect Matchings by Minimal Energies. MATCH Communications in Mathematical and in Computer Chemistry, 66, 927-942.

[21] Zhu, J. (2013) On Minimal Energies of Unicyclic Graphs with Perfect Mathching. MATCH Communications in Mathematical and in Computer Chemistry, 70, 97-118.

[22] Zhang, J. and Zhou, B. (2005) On Bicyclic Graphs with Minimal Energies. Journal of Mathematical Chemistry, 37, 423-431. https://doi.org/10.1007/s10910-004-1108-x

[23] Li, X. and Zhang, J. (2007) On Bicyclic Graphs with Maximal Energy. Linear Algebra and Its Applications, 427, 87-98. https://doi.org/10.1016/j.laa.2007.06.022 
[24] Huo, B., Ji, S., Li, X. and Shi, Y. (2011) Solution to a Conjecture on the Maximal Energy of Bipartite Bicyclic Graphs. Linear Algebra and Its Applications, 435, 804-810. https://doi.org/10.1016/j.laa.2011.02.001

[25] Ji, S. and Li, J. (2012) An Approach to the Problem of the Maximal Energy of Bicyclic Graphs. MATCH Communications in Mathematical and in Computer Chemistry, 68, 741-762.

[26] Li, S., Li, X. and Zhu, Z. (2008) On Tricyclic Graphs with Minimal Energy. MATCH Communications in Mathematical and in Computer Chemistry, 59, 397-419.

[27] Li, X., Shi, Y. and Wei, M. (2014) On a Conjecture about Tricyclic Graphs with Maximal Energy. MATCH Communications in Mathematical and in Computer Chemistry, 72, 183-214.

[28] Li, X., Mao, Y. and Liu, M. (2015) More on a Conjecture about Tricyclic Graphs with Maximal Energy. MATCH Communications in Mathematical and in Computer Chemistry, 73, 11-26. https://doi.org/10.1016/j.comcom.2015.07.003

[29] Li, X., Shi, Y. and Gutman, I. (2012) Graph Energy. Springer, New York. https://doi.org/10.1007/978-1-4614-4220-2

[30] Gutman, I. (2001) The Energy of a Graph: Old and New Results, Algebraic Combinatorics and Applications. Springer-Verlag, Berlin, 196-211.

[31] Gutman, I. and Wagner, S. (2012) The Matching Energy of a Graph. Discrete Applied Mathematics, 160, 2177-2187. https://doi.org/10.1016/j.dam.2012.06.001

[32] Aihara, J. (1976) A New Definition of Dewar-Type Resonance Energies. Journal of the American Chemical Society, 98, 2750-2758. https://doi.org/10.1021/ja00426a013

[33] Gutman, I., Milun, M. and Trinajstic, N. (1975) Topological Definition of Delocalisation Energy. MATCH Communications in Mathematical and in Computer Chemistry, 1, 171-175.

[34] Gutman, I., Milun, M. and Trinajstic, N. (1977) Graph Theory and Molecular Orbitals 19. Nonparametric Resonance Energies of Arbitrary Conjugated Systems. Journal of the American Chemical Society, 99, 1692-1704. https://doi.org/10.1021/ja00448a002

[35] Zhu, J. and Yang, J. (2018) On the Minimal Matching Energies of Unicyclic Graphs. Discrete Applied Mathematics. https://doi.org/10.1016/j.dam.2018.06.013

[36] Chen, L. and Liu, J. (2015) The Bipartite Unicyclic Graphs with the First $\lfloor(n-3) / 4\rfloor$ Largest Matching Energies. Applied Mathematics and Computation, 268, 644-656. https://doi.org/10.1016/j.amc.2015.06.115

[37] Chen, L., Liu, J. and Shi, Y. (2016) Bounds on the Matching Energy of Unicyclic Odd-Cycle Graphs. MATCH Communications in Mathematical and in Computer Chemistry, 75, 315-330.

[38] Ji, S., Li, X. and Shi, Y. (2013) Extremal Matching Energy of Bicyclic Graphs. MATCH Communications in Mathematical and in Computer Chemistry, 70, 697-706.

[39] Liu, X., Wang, L. and Xiao, P. (2018) Ordering of Bicyclic Graphs by Matching Energy. MATCH Communications in Mathematical and in Computer Chemistry, 79, 341-365.

[40] Chen, L. and Shi, Y. (2015) The Maximal Matching Energy of Tricyclic Graphs. MATCH Communications in Mathematical and in Computer Chemistry, 73, 105-119.

[41] Ji, S. and Ma, H. (2014) The Extremal Matching Energy of Graphs. Ars Combinato- 
ria, 115, 343-355.

[42] Li, S. and Yan, W. (2014) The Matching Energy of Graphs with Given Parameters. Discrete Applied Mathematics, 162, 415-420. https://doi.org/10.1016/j.dam.2013.09.014

[43] Li, H., Zhou, Y. and Su, L. (2014) Graphs with Extremal Matching Energies and Prescribed Paramaters. MATCH Communications in Mathematical and in Computer Chemistry, 72, 239-248.

[44] Wang, W. and So, W. (2015) On Minimum Matching Energy of Graphs. MATCH Communications in Mathematical and in Computer Chemistry, 74, 399-410.

[45] Xu, K., Das, K.C. and Zheng, Z. (2015) The Minimal Matching Energy of $(n, m)$ -Graphs with a Given Matching Number. MATCH Communications in Mathematical and in Computer Chemistry, 73, 93-104.

[46] Chen, L., Liu, J. and Shi, Y. (2015) Matching Energy of Unicyclic and Bicyclic Graphs with a Given Diameter. Complexity, 21, 224-238. https://doi.org/10.1002/cplx.21599

[47] Zou, L. and Li, H. (2016) The Minimum Matching Energy of Bicyclic Graphs with Given Girth. Rocky Mountain Journal of Mathematics, 46, 1275-1291. https://doi.org/10.1216/RMJ-2016-46-4-1275

[48] Shang, Y.L. (2015) The Estrada Index of Evolving Graphs. Applied Mathematics and Computation, 250, 415-423. https://doi.org/10.1016/j.amc.2014.10.129

[49] Shang, Y.L. (2015) Laplacian Estrada and Normalized Laplacian Estrada Indices of Evolving Graphs. PLoS ONE, 10, e0123426.

https://doi.org/10.1371/journal.pone.0123426 